\title{
Systemic Vascular Resistance
}

National Cancer Institute

\section{Source}

National Cancer Institute. Systemic Vascular Resistance. NCI Thesaurus. Code C119248.

The resistance to blood flow offered by all systemic vasculature, excluding pulmonary vasculature. The major determinant of SVR is arteriolar tone, but blood viscosity and vascular capacitance are also contributing factors. 